Wien klin Mag 2020 · 23:298-302 https://doi.org/10.1007/s00740-020-00372-y Online publiziert: 20. November 2020 (c) Der/die Autor(en) 2020

\section{Sirak Petros ${ }^{1,2} \cdot$ Lorenz Weidhase $^{1}$}

${ }^{1}$ Interdisziplinäre Internistische Intensivmedizin, Universitätsklinikum Leipzig, Leipzig, Deutschland

${ }^{2}$ Medizinische Klinik 1, Bereich Hämostaseologie, Universitätsklinikum Leipzig, Leipzig, Deutschland

\title{
Labordiagnostik in der Intensivmedizin
}

\section{Hintergrund}

In der Intensiv- und Notfallmedizin werden täglich zahlreiche Laborparameter angeordnet. Die Intention dieser Laborbestimmungen ist die Erfassung des Zustands des kritisch kranken Patienten sowie die Einleitung von Therapiemaßnahmen und deren Überwachung. Dabei spielt aber auch die Sorge, etwas Wichtiges am Zustand des Patienten zu übersehen, eine wesentliche Rolle. Oft entsteht der Eindruck, dass Laborwerte eine Kompensation für eine unzureichende Anamneseerhebung und klinische Untersuchung geworden sind. Zahlreiche Laboranordnungen haben keine Konsequenz in der Patientenbehandlung. Die Gründe für solche Anordnungen sind oft Unwissenheit und Unsicherheit [1].

Kritisch kranke Patienten weisen zahlreiche normabweichende Laborbefunde auf, wobei deren klinische Relevanz fragwürdig oder nicht immer eindeutig ist. Die Definition eines pathologischen Laborwerts basiert auf Normalbereichen, die aus Untersuchungen gesunder Personen erstellt worden sind. Jedoch berücksichtigt diese Definition die physiologischen Anpassungen auf einen Stress nicht; manche Laborabweichungen können lediglich Epiphänomene sein [2]. Die Grenze zwischen einer physiologischen Anpassungsreaktion und einer korrekturbedürftigen pathologischen Veränderung ist oft unscharf [3]. Die Unterscheidung zwischen den Laborpa-

Dieser Beitrag wurde in der Zeitschrift Medizinische Klinik - Intensivmedizin und Notfallmedizin 7 - (2020) 115:539-544. https://doi.org/ 10.1007/s00063-020-00730-y erstpubliziert. Zweitpublikation mit freundlicher Genehmigung der Autoren. rametern mit relevanter diagnostischer und therapeutischer Konsequenz und denen ohne einen konkreten Zweck ist ebenfalls oft verschwommen. Dies kann individuell unterschiedlich sein, wobei die Akutsituation, chronische Erkrankungen, Begleitmedikamente und genetische Faktoren eine wichtige Rolle spielen. Die Bemühungen um „Normalisierung" abweichender Parameter können im günstigen Fall zwecklos sein und im schlimmsten Fall manchmal gefährlich werden.

Eine übermäßige Laboranordnung kommt in der Akutmedizin oft vor. Nach einer Metaanalyse beträgt dies im Durchschnitt $43,9 \%$ bei der initialen Vorstellung eines Patienten [4]. In einer weiteren Studie waren Fachärzte der Auffassung, dass nur 48,7 \% der angeordneten Laborwerte essenziell waren [5]. Die Laboranordnungen bedeuten, dass mehrfache Blutentnahmen erforderlich sind, die beim kritisch Kranken zu einer iatrogenen Anämie führen [6-9] und erhebliche Kosten verursachen [4, 5, 10]. Eine übermäßige Labordiagnostik birgt schließlich auch das Risiko, von dem wesentlichen Problem abzulenken und folglich essenzielle Therapiemaßnahmen zu verzögern. Der sorgfältige Umgang mit der Anordnung und Interpretation von Laborparametern sollte daher eins der zentralen Prinzipien in der Akutmedizin sein.

\section{Präanalytische Herausforderungen}

Die Art und Weise der Blutprobengewinnung kann Einfluss auf die Laborergebnisse haben. In der Akutsituation werden Blutproben nicht selten aus einem kläg- lich funktionierenden peripher-venösen Zugang mühsam gewonnen. Die Ergebnisse solcher Entnahmen, gerade in Bezug auf eine „Point-of-Care“-Labordiagnostik (z. B. Kalium, Sauerstoffpartialdruck $\left[\mathrm{pO}_{2}\right]$, Kohlendioxidpartialdruck $\left[\mathrm{pCO}_{2}\right]$ und Laktat) und die Blutgerinnung können bei Nichtbeachtung der Präanalytik zu einer falschen Diagnose und Behandlung führen. Ähnliche Fehler können auch durch unzureichende Handhabung der Probengewinnung aus einem Zentralvenenkatheter entstehen. Auch die Transportdauer vom Patienten bis zum Labor hat bei der Analyse einiger Laborparameter einen relevanten Einfluss, wobei dieses Problem in vielen Krankenhäusern durch Etablierung des Rohrpostsystems weitgehend behoben worden ist.

\section{Pathophysiologische und klinische Herausforderung}

Eine kritische Erkrankung ist nicht nur eine diagnostische, sondern auch in großem Maß eine pathophysiologische Herausforderung. Nahezu jeder Laborparameter in der Intensivmedizin erfordert eine differenzialdiagnostische Betrachtung. Beispielsweise kann die Ursache einer Hyperlaktatämie eine übermäßig gesteigerte Glykolyse (z.B. durch Sympathikusaktivierung oder Adrenalingabe), eine Veränderung im Transportsystem zwischen Zytosol und Mitochondrien (z.B. Störung des Pyruvatdehydrogenasesystems) oder primär mitochondrial (z. B. Störung des Elektrontransportsystems oder eine Hypoxie) bedingt sein. Die Störung des Laktatshuttles, also das Zusammenspiel zwischen glykolytischen (z.B. Skelett- 
Patientenaufnahme - Erfassung der Homöostase

- Diagnosestellung

- Scores

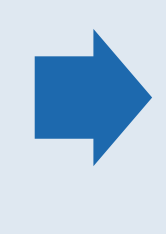

Verlauf

- Überwachung

- Diagnosestellung

- Scores

- Therapiekontrolle

Abb. 1 A Prozessabläufe in der Labordiagnostik

muskulatur) und oxidativen Zellen (z. B. Hepatozyten), kann ebenfalls zur Hyperlaktatämie führen [11]. Eine Hyperlaktatämie wird aber oft grob als Zeichen einer zellulären Hypoxie betrachtet. Diese Verallgemeinerung könnte jedoch dazu führen, dass Maßnahmen zur Korrektur einer nicht vorhandenen zellulären $\mathrm{Hy}$ poxie eingeleitet werden, was meist eine Volumengabe bedeutet.

Der kritisch kranke Patient reagiert auf seine Akutsituation durch zahlreiche physiologische Anpassungen, wodurch es sowohl zu einer Hoch- als auch Herunterregulierung zahlreicher Vorgänge kommt. Während beispielsweise einige Proteine hochreguliert werden (z.B. C-reaktives Protein), werden andere Proteine herunterreguliert (z. B. Albumin). Was die Hochregulierung angeht, ist es auch nicht hinreichend definiert, welches Ausmaß an Hochregulation als dem Stress angemessen gelten sollte. Ein Beispiel ist die Hochregulierung des Gerinnungsfaktors VIII infolge einer Inflammation oder Stresssituation. Dieses Gerinnungsprotein wird unter Stressbedingungen unterschiedlich stark freigesetzt. Das Labor orientiert sich "normal“ jedoch auf der Grundlage der Messungen an gesunden stressfreien Personen. So kann eine dem Stress unzureichend hochregulierte FVIII-Aktivität als normal angegeben werden, zumal das Labor den tatsächlichen Zustand des kritisch kranken Patienten gar nicht kennt. Die Nichtberücksichtigung dieser physiologischen Phänomene könnte dazu führen, dass pathologische Störungen übersehen oder zu spät erkannt werden.

Außerdem ist das Gleichgewicht zwischen dem Verbrauch und der Nachproduktion zellulärer und humoraler Bestandteile schwer zu definieren. Beispielsweise ist der bei Sepsis oft beobach- tete Abfall der Thrombozytenzahl das Ergebnis aus dem gesteigerten Verbrauch und der Produktion. Das Gleichgewicht zwischen Verbrauch und Produktion ist jedoch nicht nur durch das Ausmaß der auslösenden Ursache, sondern auch durch die Produktionskapazität definiert. Hierbei spielen auch Begleiterkrankungen, Medikamente, aber möglicherweise auch genetische Faktoren eine große Rolle. Solche Verschiebungen zwischen Verbrauch und Nachproduktion oder, wie bereits erwähnt, zwischen Hochund Herunterregulation beobachtet man auch im Gerinnungssystem, wodurch die Gerinnungsdiagnostik in der Intensivmedizin ohne eine konkrete klinische Fragestellung kaum sinnvoll ist.

Laborparameter, die sonst für bestimmte Erkrankungen möglicherweise diagnostisch hilfreich sind, weisen bei vielen kritischen Erkrankungen eine geringe Spezifität auf. Ein erhöhtes Serumtroponin bei kritisch kranken Patienten ohne ein akutes Koronarsyndrom mag mit einer erhöhten Sterblichkeit assoziiert sein $[12,13]$, dennoch hilft diese Erkenntnis dem Behandlungsteam wohl kaum.

Die unzureichende Antizipation der Dynamik von Laborparametern kann schließlich sowohl zu deren übermäßigen als auch unzureichenden Kontrolle führen [4].

\section{Grundanforderung an Laboranordnungen in der Akutmedizin}

Die Indikationen für die Labordiagnostik in der Intensiv- und Notfallmedizin lassen sich in 2 Hauptprozessabläufen zusammenfassen (• Abb. 1), wobei die in dem jeweiligen Ablauf erforderlichen
Laborparameter Überlappungen aufweisen.

\section{Erfassung der Homöostase und des Schweregrads der Akutsituation}

Jeder Stress hat einen Einfluss auf den zellulären Stoffwechsel und die Organfunktion. Die zeitnahe Erfassung der Homöostase während der Ersteinschätzung eines kritisch Kranken besitzt daher eine zentrale Bedeutung. Die Blutgasanalyse hat in dieser Hinsicht zu einer erheblichen Verbesserung dieser Ersteinschätzung und zur Therapiesteuerung beigetragen. Neben der eigentlichen Blutgasanalyse ermöglichen die modernen Point-of-Care-Blutgasanalysegeräte zusätzlich die Bestimmung von Natrium, Kalium, Chlorid, ionisiertem Kalzium, Glukose, Hämoglobin, Hämatokrit und Laktat. Idealerweise ist die arterielle Blutgasanalyse $\mathrm{zu}$ favorisieren, da sie auch für die Berechnung des Sequential-organ-function-assessment(SOFA)Scores benötigt wird, der z.B. in der Diagnose der Sepsis eine zentrale Rolle spielt $[14,15]$.

Neben der Blutgasanalyse sind die für Scoreberechnungen erforderlichen Laborwerte, unabhängig von der Grunderkrankung des Patienten, relevant (- Tab. 1). Diese Scores sind nicht nur für die Prognoseeinschätzung, sondern auch für die Abrechnung der intensivmedizinischen Komplexbehandlung erforderlich. Hierzu wird der Simplified-acute-physiology(SAPS)-II-Score täglich erhoben. Außerdem können diese Scores für das Benchmarking von großem Nutzen sein, um so die Qualität der einzelnen Intensivstationen vergleichen zu können.

\section{Diagnosestellung}

Die Spezifität zahlreicher Laborparameter ist in der Intensivmedizin durch das simultane Auftreten verschiedener Organinteraktionen und Schädigungen beeinträchtigt, sodass ohne konkrete Fragestellung die Interpretation schwierig ist. Bei der Anordnung von Laborparametern ist es daher sinnvoll, die Arbeitsdiagnose sowie mögliche Differenzialdia- 
gnosen strukturiert zu dokumentieren. Ein gutes Beispiel ist die Einbeziehung des SOFA-Scores bei der Diagnose der Sepsis. Der Score wird seit etwa 24 Jahren in der Intensivmedizin zur objektiven Dokumentation der Organdysfunktionen angewandt, ohne dabei zwischen chronischen und akuten Organdysfunktionen zu unterscheiden. Bei der Sepsis dagegen geht es um neue SOFA-ScorePunkte, die unter Umständen ohne Vorbefunde nicht sicher auszumachen sind. Darüber hinaus darf der SOFA-Score in der Diagnose der Sepsis nur dann verwendet werden, wenn eine Infektion bereits angenommen worden ist. Andererseits kann ein steigender SOFA-Score Anlass sein, nach einer Infektion zu fahnden.

Einige der oft als „Diagnosemarker“ missbrauchten Laborparameter sind die Entzündungsparameter. Infektion, die hier meist adressiert werden, und Inflammation, die an sich die Wirtsreaktion auf eine Noxe (z.B. Infektion, Schock oder Trauma) darstellt, sind 2 unterschiedliche Zustände. Leider werden jedoch oft die Entzündungsparameter in „Infektparameter" umgetauft und häufig angeordnet, ohne dass vorher die Arbeitsdiagnose einer Infektion konkret gestellt wurde. Beispielsweise kommt es beim kardiogenen Schock [16] oder beim akuten Leberversagen [17] zum Anstieg des Serumprokalzitonins, ohne dass eine Infektion vorliegt. Die Bedeutung dieses Befunds ist noch unklar; nach aktuellem Kenntnisstand besitzt er aber keine therapeutische Konsequenz. Die Diagnose einer Infektion basiert nicht auf den Absolutwerten verschiedener Entzündungsparameter, sondern an erster Stelle auf der Anamnese und klinischen Untersuchung. Entsprechend des klinischen Verdachts sollten die vermuteten Infektionsfoci durch bildgebende Verfahren und mikrobiologische Diagnostik validiert werden [18-20].

\section{Verlaufs- und Therapiekontrolle}

Im Lauf der Patientenversorgung sind bestimmte Laborparameter zwecks Verlaufs- und Therapiekontrolle hilfreich und erforderlich. Allerdings ist die Evidenz über ihre Aussagekraft unterschiedlich. Blutgasanalysen werden oft in der

Wien klin Mag 2020 -23:298-302 https://doi.org/10.1007/s00740-020-00372-y

(c) Der/die Autor(en) 2020

\section{S. Petros $\cdot$ L. Weidhase \\ Labordiagnostik in der Intensivmedizin}

\section{Zusammenfassung}

Die sorgfältige Anamneseerhebung und die klinische Untersuchung bleiben nach wie vor die wichtigsten diagnostischen Säulen. In der Intensiv- und Notfallmedizin werden zahlreiche Laborparameter erhoben. Die Rate an übermäßigen Laboranordnungen während der Patientenaufnahme beträgt fast $50 \%$. Oft sind die anamnestischen Angaben unzureichend, um eine gezielte Labordiagnostik vorzunehmen. Die Sorge, etwas zu übersehen, führt auch zu einer erweiterten Diagnostik. Zur Vereinfachung der Behandlungsabläufe werden oft a priori festgelegte Laborprofile benutzt, die symptombezogen angeordnet sind. Zahlreiche Laborparameter sind bei kritisch kranken Patienten außerhalb des Normbereichs. Allerdings wird der Normbereich anhand der Daten gesunder Personen definiert, der jedoch eine klare Unterscheidung zwischen Stressadaptation und klinisch relevanten korrekturbedürftigen Störungen nicht erlaubt. Die pathophysiologischen Veränderungen in vielen Körperregionen infolge der akuten Schädigung, der Reaktion des Organismus und auch der Therapiemaßnahmen führen zu Veränderungen zahlreicher Laborparameter. Ziellose Laborentnahmen tragen zur iatrogenen Anämie und zum Kostenanstieg bei. Außerdem werden die Ergebnisse solcher Entnahmen entweder kaum wahrgenommen oder können im schlimmsten Fall weitere unnötige diagnostische Schritte bzw. eine nicht gerechtfertigte Therapiemaßnahme verursachen. Die Point-of-Care-Labordiagnostik zur Einschätzung der zellulären Homöostase, die die Blutgasanalyse, ein Blutbild sowie die Bestimmung der Serumelektrolyte und des Serumlaktats beinhaltet, sowie die Laborparameter zur Berechnung der intensivmedizinisch relevanten Scores sind einheitlich erforderlich. Darüber hinaus sollte jedoch die Labordiagnostik bezogen auf konkrete klinische Fragestellung klug gewählt werden.

\section{Schlüsselwörter}

Kritische Erkrankung · Notfallmedizin .

Diagnose - Labordiagnose - Entscheidungsfindung

\section{Laboratory testing in intensive care medicine}

\section{Abstract}

Despite the tremendous technological developments in medicine, careful historytaking and clinical examination remain the cornerstones of diagnostics. Numerous laboratory tests are ordered in intensive care and emergency medicine. The rate of overutilization of these tests during initial patient admission is almost $50 \%$. Patient history may be frequently insufficient for conducting targeted laboratory testing, and concern about not overlooking a pathology also contributes to laboratory test overutilization. On the other hand, laboratory test profiles are frequently defined a priori to simplify the management process. However, these profiles are commonly based on symptoms rather than on a suspected diagnosis. Several laboratory variables are outside the normal range in critically ill patients. However, normal ranges are defined on the basis of data from healthy subjects, and these do not allow for a clear distinction between stress adaptation and clinically relevant changes that require correction. Pathophysiological changes due to the acute injury in critically ill patients and the reaction of the organism to the injury or even to the treatment itself can lead to changes in laboratory values. Untargeted laboratory tests contribute to iatrogenic anemia and increased costs. The results of such tests are either hardly noticed or, in the worst case, lead to further unnecessary diagnostic steps and unjustified therapeutic measures. Pointof-care laboratory tests, including blood gas analysis, blood count, serum electrolytes, and lactate, to assess the patient's homeostatic state and laboratory data for the relevant critical care scores are uniformly required. Beyond that, every laboratory test should be chosen wisely based on a concrete clinical question.

\section{Keywords}

Critical illness - Emergency medicine . Diagnosis · Laboratory diagnosis · Decisionmaking 


Tab. 1 Labordiagnostik in der Einschät-
zung der Homöostase und des Schwere-
grads der Akutsituation
Blutgasanalyse
Serumelektrolyte
Blutzucker
Serumlaktat
Kleines Blutbild
Serumkreatinin
Serumharnstoff
Serumbilirubin

Überwachung der hämodynamischen Therapie sowie bei extrakorporalen Organersatzverfahren benötigt (- Tab. 2). Wie oft eine Blutgasanalyse täglich erforderlich ist, hängt von der klinischen Antizipation der Dynamik und der Häufigkeit an Behandlungsbedarf ab. Dies setzt eine gute Kenntnis der Physiologie und Pathophysiologie voraus, um sowohl übermäßige als auch unzureichende Bestimmungen zu vermeiden. Laboranordnungen nach starrem Schema („Blutgasanalyse 3-stündlich“) sind sinnlos, werden oft nicht einmal wahrgenommen, bedeuten zusätzlichen Arbeitsaufwand [21] und tragen zur iatrogenen Anämie und Kostensteigerung bei.

Weitere Laborparameter zur Verlaufs- oder Therapiekontrolle sollten nach konkreten Fragestellungen und deren Sensitivität und Spezifität festgelegt werden. Entzündungsparameter können zur Kontrolle einer antiinfektiven Therapie erforderlich werden, wenn auch die Evidenz nicht einheitlich ist. Der Vorzug eines bestimmten Entzündungsparameters ist institutionell unterschiedlich, wobei auch ökonomische Aspekte eine Rolle spielen. Da die Therapiedauer bestimmter Infektionen unabhängig vom Entzündungsparameter verschieden lang sein kann (z. B. Sepsis durch Staphylococcus aureus, Endokarditis, Osteomyelitis etc.), sollte der Einsatz eines Entzündungsparameters zwecks Therapiekontrolle oder Definition der Therapiedauer individuell festgelegt werden $[18,19]$.

Die Gerinnungsdiagnostik ohne eine konkrete Frage ist in der Intensivmedizin meist nicht hilfreich. Selbst dann lassen

Tab. 2 Labordiagnostik zur Verlaufs- und
Therapiekontrolle
Blutgasanalyse
Serumelektrolyte
Serumlaktat
Kleines Blutbild
Serumkreatinin
Serumharnstoff
Serumbilirubin
Entzündungsparameter
Zielgerichtete Gerinnungsdiagnostik
Medikamentenspiegel

4. Durch die Aktivierung der Entzündungskaskade kann, z. B. bei therapeutischer Antikoagulation mittels unfraktionierten Heparins, die Steuerung umständlich werden.

5. Bei Medikamenten mit geringer therapeutischer Breite drohen ohne Kontrolle der Wirkspiegel Unterdosierungen mit unzureichender Wirkung oder Überdosierung mit toxischen Effekten.

\section{Korrelation mit dem klinischen Szenario}

sich nicht alle Fragen durch die verfügbaren Labormethoden beantworten. Die Kontrolle einer therapeutischen Antikoagulation ist Standard, wobei die Häufigkeit der Kontrollen individuell unterschiedlich ist. Gerade bei kritisch kranken Patienten mit massiver Inflammation kann die therapeutische Antikoagulation mittels unfraktionierten Heparins Probleme bereiten.

Das Ziel der Gerinnungstherapie im Rahmen einer Blutung ist nicht die Normalisierung der Gerinnungsparameter, sondern die Blutstillung. Die für eine Blutstillung erforderliche Höhe der Gerinnungsparameter ist dabei individuell unterschiedlich. Daher lassen einzelne Gerinnungsparameter Rückschlüsse auf den hämostatischen Zustand des kritisch kranken Patienten nicht ohne Weiteres $\mathrm{zu}$.

Die Spiegelbestimmungen bestimmter Antiinfektiva sind zunehmend relevant geworden. Auch Immunsuppressiva und Antikonvulsiva sollten regelmäßig kontrolliert werden. Diese Kontrollen sind aus folgenden Gründen erforderlich: 1. Der häufig veränderte Flüssigkeitshaushalt, die Kapillardurchlässigkeit sowie Hoch- oder Unterregulation von Organfunktionen beim kritisch Kranken machen die Dosierung von Medikamenten schwierig.

2. Die Dynamik der Organfunktion (besonders renal und hepatisch) des kritisch Kranken erfordert eine zeitgerechte Dosisanpassung.

3. Die intensivmedizinisch oft unvermeidbare Polypharmazie kann zu Medikamenteninteraktionen führen.
Nicht zuletzt müssen Laborwerte stets im Kontext des klinischen Szenarios betrachtet werden. Präanalytische Fehler bei Probengewinnung oder Transport können dazu führen, dass die Ergebnisse falsch sind. Beispielsweise liefert eine hämolytische Blutprobe einen falsch hohen Laktatwert; eine Blutprobe mit Luftblasen kann $\mathrm{zu}$ falschen $\mathrm{pO}_{2}$ - und $\mathrm{pCO}_{2-}$ Werten bei der Blutgasanalyse führen. Eine nicht korrekt durchgeführte Vorspülung des Zentralvenenkatheters kann dazu führen, dass diverse Laborwerte, etwa Blutbild, Gerinnungsparameter oder Elektrolyte, falsch sein können. Da Blutentnahmen meist durch Pflegekräfte oder Studenten vorgenommen werden, sollten Intensivmediziner durch regelmäßige Schulungen für die Optimierung der präanalytischen Qualität sorgen.

\section{Zielgerichtete Laboranordnung}

Um die Aussagefähigkeit von Laborwerten zu optimieren, Verlust an Patientenblut zu minieren und vermeidbare Kosten $\mathrm{zu}$ senken, ist es erforderlich, Anordnungen regelmäßig kritisch zu überprüfen. Dazu können validierte interne Richtlinien [22], ein Audit und Feedback [23-25], Fortbildungen [26] und der Einsatz künstlicher Intelligenz [27] sinnvoll sein.

\section{Fazit für die Praxis}

\footnotetext{
- Laboranordnungen werden in der Intensiv- und Notfallmedizin häufig vorgenommen. Diese Anordnungen sollten stets einen konkreten klinischen Bezug haben. Laborpara-
} 
meter dürfen nicht als Kompensation für eine unzureichende klinische Evaluation missbraucht werden.

- Übermäßige Laboranforderungen kommen gerade bei der initialen Einschätzung kritisch kranker Patienten sehr oft vor. Diese verursachen eine iatrogene Anämie, hohe Kosten, falsche Diagnosen sowie sinnlose oder gar gefährliche Therapiemaßnahmen.

- Die Hauptindikationen für Laboranordnungen in der Akutmedizin sind die Erfassung und Kontrolle der Homöostase, die Diagnosestellung, die Scoreberechnung und die Verlaufsund Therapiekontrolle.

- Präanalytische Faktoren, die fehlende scharfe Abgrenzung zwischen normalen und pathologischen Bereichen sowie zwischen physiologischer Anpassung und therapierelevanter Störung müssen bei der Interpretation und Einleitung einer Behandlungsmaßnahme berücksichtigt werden.

\section{Korrespondenzadresse}

\section{Prof. Dr. Sirak Petros}

Interdisziplinäre Internistische Intensivmedizin, Universitätsklinikum Leipzig Liebigstr. 20, 04103 Leipzig, Deutschland sirak.petros@medizin.uni-leipzig.de

Funding. Open Access funding enabled and organized by Projekt DEAL.

\section{Einhaltung ethischer Richtlinien}

Interessenkonflikt. S. Petros und L. Weidhase geben an, dass kein Interessenkonflikt besteht.

Für diesen Beitrag wurden von den Autoren keine Studien an Menschen oder Tieren durchgeführt. Für die aufgeführten Studien gelten die jeweils dort angegebenen ethischen Richtlinien.

Open Access Dieser Artikel wird unter der Creative Commons Namensnennung 4.0 International Lizenz veröffentlicht, welche die Nutzung, Vervielfältigung, Bearbeitung, Verbreitung und Wiedergabe in jeglichem Medium und Format erlaubt, sofern Sie den/die ursprünglichen Autor(en) und die Quelle ordnungsgemäß nennen, einen Link zur Creative Commons Lizenz beifügen und angeben, ob Änderungen vorgenommen wurden.

Die in diesem Artikel enthaltenen Bilder und sonstiges Drittmaterial unterliegen ebenfalls der genannten
Creative Commons Lizenz, sofern sich aus der Abbildungslegende nichts anderes ergibt. Sofern das betreffende Material nicht unter der genannten Creative Commons Lizenz steht und die betreffende Handlung nicht nach gesetzlichen Vorschriften erlaubt ist, ist für die oben aufgeführten Weiterverwendungen des $\mathrm{Ma}$ terials die Einwilligung des jeweiligen Rechteinhabers einzuholen.

Weitere Details zur Lizenz entnehmen Sie bitte der Lizenzinformation auf http://creativecommons.org/ licenses/by/4.0/deed.de.

\section{Literatur}

1. Vrijsen BEL, Naaktgeboren CA, Vos LM et al (2020) Inappropriate laboratory testing in internal medicine inpatients: prevalence, causes and interventions. Ann Med Surg 51:48-53

2. Tyler PD, Du H, Feng M et al (2018) Assessment of intensive care unit laboratory values that differ from reference ranges and association with patient mortality and length of stay. JAMA Netw Open 1:e184521

3. Anderson WG, Cimino JW, Ernecoff NC et al (2015) A multicenter study of key stakeholders' perspectives on communicating with surrogates about prognosis in intensive care units. Ann Am Thorac Soc 12:142-152

4. Zhi M, Ding EL, Theisen-Toupal J et al (2013) The landscape of inappropriate laboratory testing: a 15-year meta-analysis. Plos One 8:e78962

5. Mikhaeil M, Day AG, Ilan R (2017) Non-essential blood tests in the intensive care unit: a prospective observational study. Can J Anaesth 64:290-295

6. Napolitano LM (2004) Scope of the problem: epidemiology of anemia and use of blood transfusions in critical care. Crit Care 8(Suppl 2):1-8

7. Ullman AJ, Keogh S, Coyer F et al (2016) 'True blood' the critical care story: an audit of blood sampling practice across three adult, paediatric and neonatal intensive care settings. Aust Crit Care 29:90-95

8. Jakacka N, Snarski E, Mekuria S (2016) Prevention of iatrogenic anemia in critical and neonatal care. Adv Clin Exp Med 25:191-197

9. Whitehead NS, Williams LO, Meleth S et al (2019) Interventions to prevent iatrogenic anemia: a laboratory medicine best practices systematic review. Crit Care 23:278

10. Bindraban RS, Ten Berg MJ, Naaktgeboren CA et al (2018) Reducing testutilization in hospital settings: a narrative review. Ann Lab Med 38:402-412

11. Brooks GA (2018) The science and translation of lactate shuttle theory. Cell Metab 27:757-785

12. Alatassi A, Habbal M, Tamim H et al (2018) Association between troponin-I levels and outcome in critically ill patients admitted to noncardiac intensive care unit with high prevalence of cardiovascular risk factors. BMC Anesthesiol 18:54

13. Lim W, Qushmaq I, Devereaux PJ et al (2006) Elevated cardiac troponin measurements in critically ill patients. Arch Intern Med 166:2446-2454

14. Vincent JL, Moreno R, Takala J et al (1996) The SOFA (Sepsis-related Organ Failure Assessment) score to describe organ dysfunction/failure. On behalf of the Working Group on Sepsis-Related Problems of the European Society of Intensive Care Medicine. Intensive Care Med 22:707-710

15. Singer M, Deutschman CS, Seymour CW et al (2016) The third international consensus definitions for sepsis and septic shock (sepsis-3). JAMA 315:801-810

16. Picariello C, Lazzeri C, Chiostri M et al (2009) Procalcitonin in patients with acute coronary syndromes and cardiogenic shock submitted to percutaneous coronary intervention. Intern Emerg Med 4:403-408

17. Rule JA, Hynan LS, Attar N et al (2015) Procalcitonin identifies cell injury, not bacterial infection, in acute liver failure. PLoSONE 10:e138566

18. Rhodes A, Evans LE, Alhazzani W et al (2017) Surviving sepsis campaign: international guidelines for management of sepsis and septic shock: 2016 . Intensive Care Med 43:304-377

19. BrunkhorstFM, Weigand MA, Pletz Metal (2020)S3 guideline sepsis-prevention, diagnosis, treatment, and aftercare: summary of the strong recommendations. Med Klin Intensivmed Notfmed 115:178-188

20. Calandra T, Cohen J (2005) The international sepsis forum consensus conference on definitions of infection in the intensive care unit. Crit Care Med 33:1538-1548

21. Artis KA, Bordley J, Mohan V et al (2019) Data omission by physician trainees on ICU rounds. Crit Care Med 47:403-409

22. Prat G, Lefèvre M, Nowak E et al (2009) Impact of clinical guidelines to improve appropriateness of laboratory tests and chest radiographs. Intensive Care Med 35:1047-1053

23. Foster M, Presseau J, Mccleary N et al (2020) Audit and feedback to improve laboratory test and transfusion ordering in critical care: a systematic review. Implement Sci 15:46

24. Kleinpell RM, Farmer JC, Pastores SM (2018) Reducing unnecessary testing in the intensive care unit by choosing wisely. Acute Crit Care 33:1-6

25. Minerowicz C, Abel N, Hunter Ket al (2015) Impact of weekly feedback on test ordering patterns. Am J Manag Care 21:763-768

26. Harb R, Hajdasz D, Landry MLet al (2019) Improving laboratory test utilisation at the multihospital Yale New Haven Health System. BMJ Open Qual 8:e689

27. Cismondi F, Celi LA, Fialho AS et al (2013) Reducing unnecessary lab testing in the ICU with artificial intelligence. Int J Med Inform 82:345-358 